\title{
Improving Fecal Occult Blood Testing Compliance Using a Mailed Educational Reminder
}

\author{
Jeffrey K. Lee, $M D^{7}$, Veronica Reis, $P h D^{2}$, Shanglei Liu, $B S^{7}$, Lorraine Conn, $M T^{2}$, \\ Erik J. Groessl, $P h D^{3}$, Theodore G. Ganiats, $M D^{3}$, and Samuel B. Ho, MD ${ }^{1,4}$
}

'Department of Medicine, VA San Diego Healthcare System and University of California, San Diego, CA, USA; '2aboratory Medicine, VA San Diego Healthcare System and University of California, San Diego, CA, USA; ${ }^{3}$ Family Medicine, VA San Diego Healthcare System and University of California, San Diego, CA, USA; ${ }^{4}$ Gastroenterology (111D), VA San Diego Healthcare System, San Diego, CA, USA.

BACKGROUND: Colorectal cancer (CRC) is one of the leading causes of cancer-related deaths in the United States. Randomized controlled trials have shown that annual screening fecal occult blood testing (FOBT) reduces $\mathrm{CRC}$ mortality and incidence. However, patient compliance with FOBT is low.

OBJECTIVE: To determine whether a mailed educational reminder increases FOBT card return rates and to examine predictors of FOBT compliance.

DESIGN: Blinded, randomized, controlled trial at the Veteran Affairs Medical Center, San Diego, California.

PATIENTS: Seven hundred and seventy-five consecutive patients $\geq 50$ years of age referred by their primary care physicians for FOBT.

INTERVENTION: Patients were randomly assigned to the usual care group or the intervention group. Ten days after picking up the FOBT cards, a 1-page reminder with information related to CRC screening was mailed to the intervention group only.

MEASUREMENTS: The primary outcome was proportion of returned FOBT cards after 6 months. Patient demographic, clinical characteristics and prior FOBT completed were collected for multivariate regression analysis.

RESULTS: At 6 months after card distribution, 64.6\% of patients in the intervention group returned cards compared with $48.4 \%$ in the control group $(\mathrm{P}<0.001)$. Patients who received a mailed reminder (OR 2.02; 95\% CI: $1.48-2.74)$ or have a prior history of returning the FOBT cards (OR 1.87; 95\% CI: 1.29-2.70) were more likely to return the FOBT cards. Patients with current or

Electronic supplementary material The online version of this article (doi:10.1007/s11606-009-1087-5) contains supplementary material, which is available to authorized users.

ClinicalTrials.gov registration number: NCT00534053

Funding: There was no outside funding for this project. All resources were provided by the VA San Diego Healthcare System, San Diego, California

Project was accepted and presented as a poster presentation at Digestive Disease Week in San Diego, California on May 18, 2008

Received November 19, 2008

Revised May 20, 2009

Accepted July 16, 2009

Published online September 23, 2009 recent illicit drug use were less likely to return the FOBT cards (OR 0.26; 95\% CI: 0.13-0.50).

CONCLUSION: A simple mailed educational reminder significantly increases compliance with FOBT for CRC screening.

KEY WORDS: reminder; colorectal cancer; screening; FOBT.

J Gen Intern Med 24(11):1192-7

DOI: $10.1007 / \mathrm{s} 11606-009-1087-5$

(C) Society of General Internal Medicine 2009

\section{INTRODUCTION}

Colorectal cancer (CRC) is one of the leading causes of cancerrelated deaths in the United States and a common cause of morbidity and mortality worldwide ${ }^{1}$. In 2008, about 148,000 new cases of CRC will be diagnosed, and about 50,000 people will die from this disease ${ }^{2}$. Several randomized controlled trials have shown evidence for the effectiveness of fecal occult blood testing (FOBT) in reducing CRC mortality by using samples from three successive stools ${ }^{3,4}$.

National efforts have been made to increase awareness of CRC screening over the past several years. Recently, the United States Preventive Services Task Force (USPSTF) and National Comprehensive Cancer Network (NCCN) recommended all individuals within age 50 to 75 years, who are at average risk for CRC, to use one of the following methods for CRC screening: an annual high sensitivity FOBT, a flexible sigmoidoscopy every 5 years, or a colonoscopy every 10 years $^{5,6}$. A Veteran Affairs (VA) directive based on previous US Multi Society Task Force and USPSTF guidelines ${ }^{7,8}$ recommended screening of all eligible patients using any one of five methods, including annual FOBT testing, flexible sigmoidoscopy every five years, the combination of annual FOBT and every five year flexible sigmoidoscopy, double contrast barium enema every 5 years, or colonoscopy alone every 10 years. Despite these recommendations and guidelines, CRC screening rates remain low. As of 2006, almost $40 \%$ of adults age 50 years or older were not up-to-date with CRC screening ${ }^{2}$. Numerous studies have shown that patient compliance in CRC screening programs and FOBT card return rates are suboptimal ${ }^{9-12}$. As a result, interest in new effective and inexpensive interventions remains high.

One method of improving compliance with screening guidelines is a patient-oriented reminder. Several studies have shown that mailed reminders can be effective, efficient, and 
inexpensive in improving cervical and breast cancer screening rates $^{13-15}$. However, few studies have evaluated whether any type of patient reminder can improve FOBT card return rates for CRC screening ${ }^{9}$. We conducted a double-blind, randomized controlled trial to examine whether a mailed educational reminder would increase FOBT card return rates. Our secondary objective was to identify demographic and clinical characteristics associated with FOBT compliance.

\section{METHODS}

\section{Design Overview}

To test the effect of a mailed educational reminder on increasing FOBT card return rate for CRC screening, we conducted a double-blind, randomized controlled trial in a U.S. veteran patient population. Figure 1 shows a flow diagram of our study design. Patients meeting inclusion criteria were randomly assigned to receive either usual care or usual care with a mailed educational reminder (intervention). The clinical research associate mailed all educational reminders 10 days after the patients received their FOBT cards. Patients were given 6 months to return the FOBT cards. The Institutional Review Board (IRB) of the University of California, San Diego (UCSD) and the Research and Development Committee of the VA San Diego Healthcare System approved the study.

\section{Study Setting and Population}

We evaluated patients from the VA Medical Center primary care clinics in San Diego and Vista between June 1 and September 9, 2007. The study included asymptomatic men and women age 50 years or older who agreed to screening and received FOBT card kits with a postage paid return envelope. Under usual care, primary care physicians (PCP) entered a computerized order for FOBT testing, and then patients were instructed by the PCP to pick up FOBT cards for CRC screening from the laboratory and return them for analysis. Patients were excluded from this study if they were less than 50 years of age, were currently on an inpatient unit, or refused to undergo any routine CRC screening. CRC screening was performed in primary care clinics in accordance with VHA Directive 2007-004 CRC Screening (wwwl.va.gov/cancer/ docs/VHA_Colorectal_Cancer_Screening_2007-004.DOC), based on recommendations from the US Multi Society Task Force (2003) and the US Preventive Services Task Force $(2002)^{7,8}$. The decision regarding which screening test to choose is dependent on patient and physician choice and local resource availability. We did not exclude any patients over the age of 75 as recommended by the USPSTF in 2008, because our study was performed prior to the 2008 guidelines $^{5}$.

\section{Randomization and Intervention}

Using a random-number generator, the clinical research associate randomly and sequentially assigned patients who met inclusion criteria, without blocking or stratification, to usual care (control) group versus an intervention group (mailed educational reminder). The randomization process and group assignment occurred after the chart review, ensuring that members of the study team abstracting data from the medical charts were blinded to the randomization results and the FOBT card return status.

The mailed educational reminder consisted of an $8.5 \times 11$ inch paper folded in thirds, personalized, sealed, and sent to the subjects' home address 10 days after the patients were given their FOBT cards by the clinical laboratory (Online appendix) . The content of the letter incorporated both a reminder and educational element based on a similar study using a mailed educational reminder for colonoscopy screening ${ }^{16}$. The mailed letter was one-sided, written at an eighth-grade reading level ${ }^{17}$, and contained a reminder to return their FOBT cards on the top portion of the letter. The middle portion of the letter

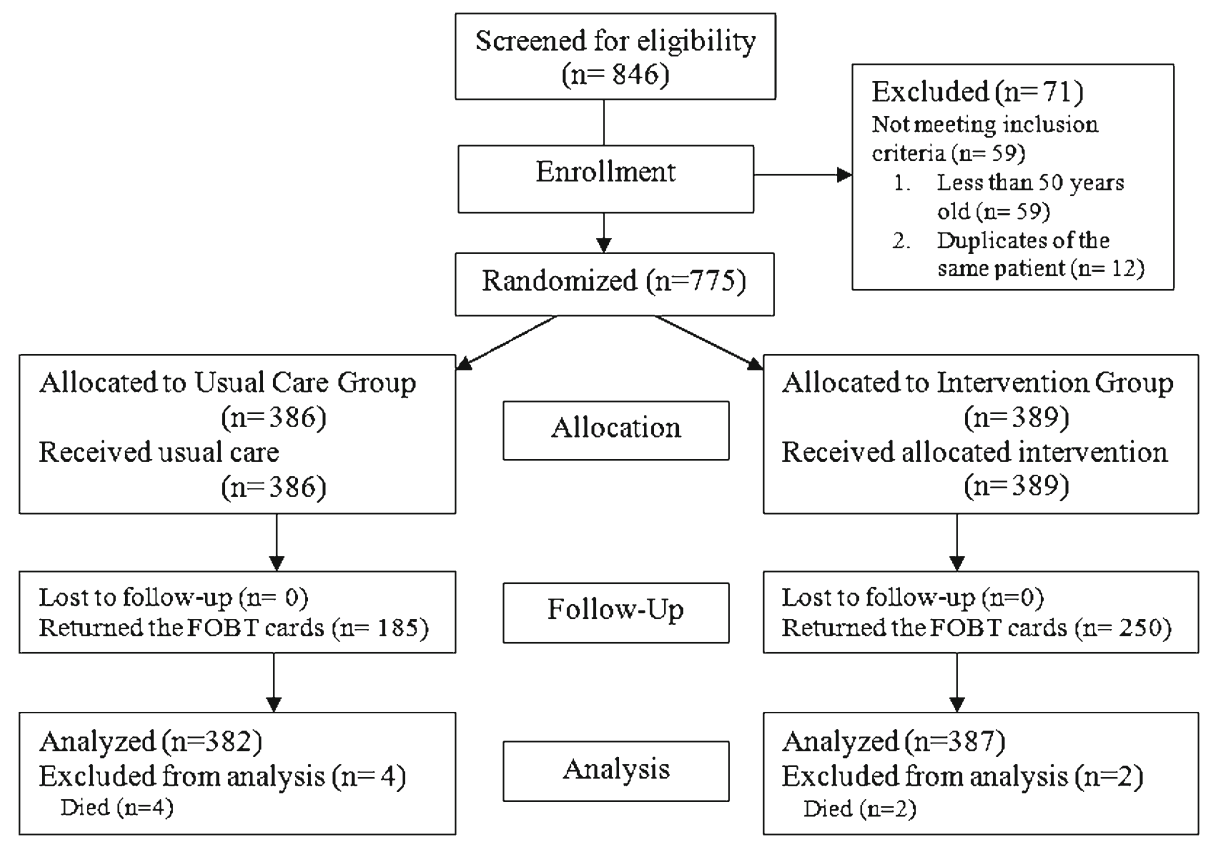

Figure 1. Flow Diagram through the study. CRC: Colorectal Cancer. 
had several statements regarding the risk of developing CRC, who is affected by the disease, and the benefits of getting screened. On the bottom third of the letter was a quote from a United States Veteran colon cancer survivor, who emphasized the importance of colorectal cancer screening. Lastly, the reminder contained a direct 24-hour, 7-day a week, VA laboratory phone number for any questions about the FOBT. The IRB at our institution granted exemption of informed consent because of the minimal risk associated with the mailed reminder and a guarantee of at least usual care for all patients.

\section{Study Outcomes}

The primary outcome of this study was the proportion of patients who returned the FOBT cards within 6 months. Our outcome measure was based on chart review by a clinical research assistant who was blinded to the patients' trial arm assignments. If a mailed educational reminder was returned by the post office as undeliverable, no further mailings were sent; however, the patient was retained in the sample for an intention-to-treat analysis.

Our secondary outcome was to identify factors associated with successfully returning FOBT cards. Using administrative databases and the VA's electronic medical record database, we retrospectively collected information on all patients who received FOBT cards and were enrolled in our study. Study members who performed the chart review were blinded to both trial assignment and FOBT card return status. A systematic method of data abstraction was used to collect the following information for each patient: age, gender, marital status, race/ ethnicity, median household income by postal code of residence, number of other scheduled and kept VA appointments for the 1-year period prior to obtaining FOBT cards, prior number of FOBT cards returned in the past, current warfarin use, history of hepatitis C or HIV, family or personal history of $\mathrm{CRC}$, history of anxiety, mood, or psychotic disorders, current or recent use of illicit drugs, and current use of tobacco or alcohol. Patients were given a diagnosis of mood, anxiety, or psychotic disorder if they were currently taking psychiatric medications, were given a corresponding ICD-9 code, or were previously diagnosed by their psychiatrist, primary care physician, or psychologist. Current or recent use of illicit drugs (marijuana, cocaine, amphetamines, opiates, and heroin) was defined as any documented record of illicit drug use or positive urine toxicology 6 months before or after picking up their FOBT cards from the laboratory.

\section{Statistical Analysis}

In determining our sample size, we hypothesized that patients in the intervention group would have at least $10 \%$ greater FOBT card return rate than patients in the usual care group. We estimated that compliance in the control group would be about $50 \%$, based on a $51.3 \%$ rate described in a similar study of a similar population ${ }^{11}$. To test our hypothesis, the target sample size was 792 patients (or 396 in each group) using a two-sided chi-square test with $80 \%$ power and alpha value set at 0.05 .

Subjects in the control group were compared with subjects in the intervention group. Comparisons for continuous variables and categorical variables were made using the Student t test and chi square analysis, respectively. The Wilcoxon rank sum test was used when the normal distribution assumption was not met for a continuous variable.

We used univariate and multivariate logistic regression analysis to determine predictors of compliance with FOBT. Univariate predictors with a $P$ value less than 0.05 were entered into a multivariate forward logistic regression model to generate adjusted odds ratios (OR) with 95\% confidence intervals (CI). Each independent variable retained in the multiple logistic regression model had a significance level of $P<0$.05. All statistical analyses were performed using SPSS software version 16.0 for Macintosh (SPSS, Chicago, IL).

\section{RESULTS}

\section{Study Participants}

Among 846 patients who received FOBT cards for CRC screening during our 3-month enrollment period, 775 were determined eligible and were randomized to receive either a mailed educational reminder or usual care (Fig. 1). Seventyone patients were excluded either due to their age (59 patients were less than 50 years old) or duplicate referral (12 patients). Six patients died within 6 months after receiving their FOBT cards and were excluded from the final analysis. Thus, our final sample size for analysis was 769 patients. The baseline demographic and clinical characteristics were well matched in both the control and intervention groups (Table 1). The mean age of our study participants was 63.1 years $(S D=9.6)$. The majority of our study participants were male (96.3\%), Caucasian (72.8\%), and not married (56.6\%); the largest minority group in the cohort was African-Americans (11.7\%). Many members of the cohort were currently drinking alcohol (45.0\%) and smoking tobacco $(27.6 \%)$, but only a few were noted to be currently or recently using illicit drugs (6.5\%). Major psychiatric disorders present in our cohort were anxiety disorders (11.3\%) and mood disorders (28.9\%).

\section{Primary Outcome}

Overall, 435 of the 769 participants (56.6\%) returned the FOBT cards within 6 months. The proportion of patients who returned the FOBT cards was significantly higher in the mailed reminder group than those who did not receive a reminder (64.6\% vs. $48.4 \%$; $\mathrm{P}<0.001)$. At 6 months after card distribution, the FOBT card return rate was 16.2 percentage points greater in the intervention group than in the control group (Fig. 2). This represents a $33.5 \%$ increase in the FOBT card return rate. As shown in Figure 2, more than 90\% of our participants returned their FOBT cards within 60 days of receiving the test from the laboratory.

\section{Multivariate Analysis}

Demographics, clinical characteristics, percentage of other VA appointments kept, and number of prior FOBT completed in the past were compared among patients who were compliant and non-compliant with FOBT as shown in Table 2. Patients who received a mailed reminder, older age group $(\geq 70$ years versus 50-59 years), percentage of other VA appointments kept, prior FOBT completed in the past, history of mood 
Table 1. Baseline Demographics and Clinical Characteristics

\begin{tabular}{|c|c|c|}
\hline Characteristics & $\begin{array}{l}\text { Usual care } \\
(n=382)\end{array}$ & $\begin{array}{l}\text { Intervention } \\
\text { group }(n=387)\end{array}$ \\
\hline Mean age, years(SD) & $63.1(9.6)$ & $62.9(9.6)$ \\
\hline Men, n (\%) & $367(96.1)$ & $374(96.6)$ \\
\hline \multicolumn{3}{|l|}{ Race / Ethnicity, n (\%) } \\
\hline White & 277(72.5) & 283(73.1) \\
\hline Black & $40(10.5)$ & $50(12.9)$ \\
\hline Hispanic & $31(8.1)$ & $27(7.0)$ \\
\hline Other & $34(9.9)$ & $27(7.0)$ \\
\hline \multicolumn{3}{|l|}{ Marital status, n (\%) } \\
\hline Not married & $221(57.9)$ & $214(55.3)$ \\
\hline Married & $161(42.1)$ & 173(44.7) \\
\hline Family history of CRC, n (\%) & 14(3.7) & $21(5.4)$ \\
\hline Personal history of CRC, n (\%) & $10(2.6)$ & $13(3.4)$ \\
\hline \multicolumn{3}{|l|}{ No. of prior FOBT, $\mathrm{n}(\%)$} \\
\hline None & 202(52.9) & $182(47.0)$ \\
\hline 1 & $81(21.2)$ & 106(27.4) \\
\hline 2 & $43(11.3)$ & $47(12.1)$ \\
\hline $3+$ & $56(14.7)$ & $52(13.4)$ \\
\hline \multicolumn{3}{|l|}{$\begin{array}{l}\text { Median income by postal code of } \\
\text { residence }\end{array}$} \\
\hline$\leq \$ 29,999$ & $32(9.1)$ & $46(12.8)$ \\
\hline$\$ 30,000-\$ 39,999$ & $61(17.4)$ & $50(14.0)$ \\
\hline$\$ 40,000-\$ 49,999$ & $115(32.9)$ & $111(31.0)$ \\
\hline$\$ 50,000-\$ 59,999$ & $43(12.3)$ & 64(17.9) \\
\hline$\$ 60,000-\$ 69,999$ & $63(18.0)$ & $53(14.8)$ \\
\hline$\geq \$ 70,000$ & $36(10.3)$ & 34(9.5) \\
\hline $\begin{array}{l}\text { Mean percentage of other VA } \\
\text { appointments kept, mean (SD) }\end{array}$ & $90.8(13.7)$ & $91.9(26.8)$ \\
\hline \multicolumn{3}{|l|}{ Psychiatric disease, $\mathrm{n}(\%)$} \\
\hline Anxiety disorder & $42(11.0)$ & $45(11.6)$ \\
\hline Mood disorder & $98(25.7)$ & $124(32.0)$ \\
\hline Psychotic disorder & $11(2.9)$ & $11(2.8)$ \\
\hline Current illicit drug use, n (\%) & $24(6.3)$ & $26(6.7)$ \\
\hline Current alcohol use, n (\%) & 177(46.3) & 169(43.7) \\
\hline Current tobacco use, n (\%) & 95(24.9) & $117(30.2)$ \\
\hline Hepatitis C & $31(8.1)$ & $33(8.5)$ \\
\hline HIV & $12(3.1)$ & $10(2.6)$ \\
\hline Current warfarin use, n (\%) & $28(7.3)$ & $22(5.7)$ \\
\hline
\end{tabular}

CRC: colorectal cancer

VA: Veteran Affairs

HIV: human immunodeficiency virus

disorder, and current or recent tobacco or illicit drug use were statistically significant in univariate analysis. Multivariate logistic regression analysis (Table 3 ) showed that receiving a mailed reminder (OR 2.02; 95\% CI: 1.48-2.74) and prior history of FOBT completion (OR 1.87; 95\% CI: 1.29-2.70) were

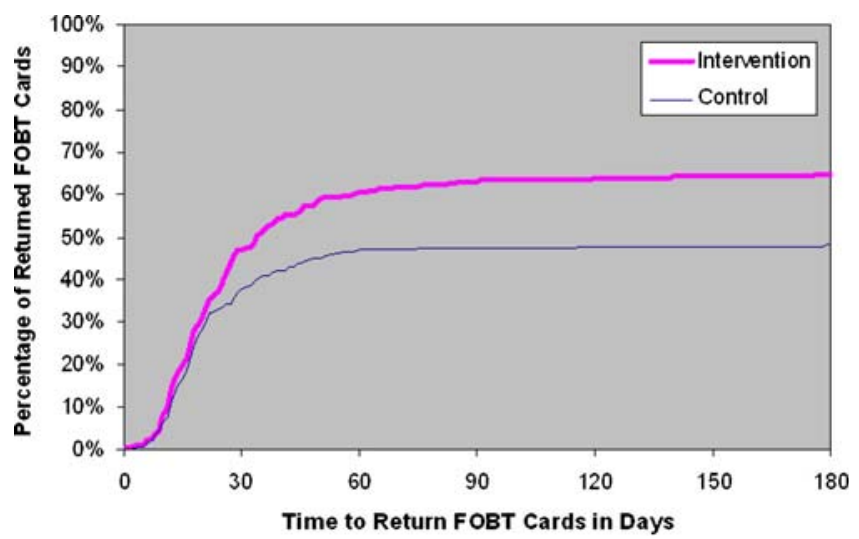

Figure 2. Cumulative adherence over time by study. FOBT: Fecal Occult Blood Test.
Table 2. Univariate Analysis of Predictors of FOBT Compliance

\begin{tabular}{|c|c|c|}
\hline & $\begin{array}{l}\text { Univariate } \\
\text { odds ratio }\end{array}$ & $\begin{array}{l}95 \% \\
\text { confidence } \\
\text { interval }\end{array}$ \\
\hline Received mailed reminder & 1.94 & $1.45-2.60$ \\
\hline \multicolumn{3}{|l|}{ Age group } \\
\hline $60-69$ versus $50-59$ & 1.38 & $0.99-1.91$ \\
\hline$\geq 70$ versus $50-59$ & 1.81 & $1.25-2.63$ \\
\hline$\%$ of VA appt. kept (per \%) & 1.01 & $1.00-1.02$ \\
\hline Male gender & 0.72 & $0.33-1.57$ \\
\hline \multicolumn{3}{|l|}{ Race group } \\
\hline Black versus white & 1.08 & $0.69-1.70$ \\
\hline Hispanic versus white & 0.66 & $0.38-1.13$ \\
\hline Other versus white & 1.09 & $0.64-1.86$ \\
\hline Married & 1.19 & $0.89-1.59$ \\
\hline Family history of CRC & 1.32 & $0.65-2.65$ \\
\hline Personal history of CRC & 1.78 & $0.73-4.40$ \\
\hline \multicolumn{3}{|l|}{ Prior FOBT returned group } \\
\hline 1 versus 0 & 1.95 & $1.36-2.78$ \\
\hline 2 versus 0 & 3.85 & $2.29-6.48$ \\
\hline$\geq 3$ versus 0 & 3.93 & $2.42-6.38$ \\
\hline \multicolumn{3}{|c|}{ Median household income by postal code } \\
\hline$\$ 30,000-39,999$ vs. $\leq \$ 29,999$ & 1.20 & $0.67-2.15$ \\
\hline$\$ 40,000-49,999$ vs. $\leq \$ 29,999$ & 1.16 & $0.69-1.93$ \\
\hline$\$ 50,000-59,999$ vs. $\leq \$ 29,999$ & 1.59 & $0.88-2.88$ \\
\hline$\$ 60,000-69,999$ vs. $\leq \$ 29,999$ & 1.61 & $0.90-2.89$ \\
\hline$\geq \$ 70,000$ vs. $\leq \$ 29,999$ & 1.13 & $0.59-2.15$ \\
\hline Anxiety disorder & 1.29 & $0.82-2.05$ \\
\hline Mood disorder & 0.72 & $0.53-0.99$ \\
\hline Psychotic disorder & 0.63 & $0.27-1.48$ \\
\hline Current or recent illicit drug use & 0.28 & $0.15-0.52$ \\
\hline Current alcohol use & 0.96 & $0.72-1.28$ \\
\hline Current tobacco use & 0.64 & $0.47-0.88$ \\
\hline Hepatitis C & 0.80 & $0.48-1.34$ \\
\hline HIV & 0.76 & $0.33-1.78$ \\
\hline Current warfarin use & 1.16 & $0.65-2.09$ \\
\hline
\end{tabular}

CRC: colorectal cancer

VA: Veteran Affairs

HIV: human immunodeficiency virus

significant predictors of compliance with FOBT. Current or recent use of illicit drugs (OR 0.26; 95\% CI: 0.13-0.50) was a significant predictor of non-compliance. The data presented in Table 3 also show that the odds of compliance increases with each number of prior FOBT completed. Compliance rates were significantly higher for those who completed one (OR 1.87), two (OR 3.89), or three or more (OR 4.05) FOBT kits in the past compared with patients who had never returned a FOBT kit.

Table 3. Multiple Logistic Regression Model of Predictors of FOBT Compliance

\begin{tabular}{lll}
\hline \hline & $\begin{array}{l}\text { Adjusted } \\
\text { odds ratio }\end{array}$ & $\begin{array}{l}\text { 95\% confidence } \\
\text { interval }\end{array}$ \\
\hline $\begin{array}{l}\text { Received a mailed reminder } \\
\text { Prior FOBT returned group }\end{array}$ & 2.02 & $1.48-2.74$ \\
$\quad$ 1 versus 0 & 1.87 & $1.29-2.70$ \\
2 versus 0 & 3.89 & $2.29-6.63$ \\
$\geq 3$ versus 0 & 4.05 & $2.46-6.66$ \\
Current or recent illicit drug use & 0.26 & $0.13-0.50$ \\
Age group & & \\
60-69 versus 50-59 & 1.08 & $0.76-1.54$ \\
$\quad 70$ versus 50-59 & 1.18 & $0.78-1.78$ \\
\% of VA appt. kept (per \%) & 1.03 & $0.99-1.01$ \\
Mood disorder & 0.78 & $0.56-1.09$ \\
Current tobacco use & 0.80 & $0.57-1.14$ \\
\hline
\end{tabular}

FOBT: fecal occult blood test 
Patients who received a mailed reminder were 2.02 times more likely to return the FOBT than those patients who did not receive a mailed reminder. Patients who were currently or recently using illicit drugs were $74 \%$ less likely to return the FOBT kits compared to non-illicit drug users. We repeated our analysis removing the intervention as a covariate in the multivariable model and none of the results were significantly altered. No patient characteristics were found to modify the effect of the mailed reminder on FOBT card return rate.

\section{DISCUSSION}

Our blinded, randomized, controlled study showed that a simple, low cost mailed educational reminder resulted in an absolute 16.2 percentage point higher FOBT card return rate compared with usual care $(64.4 \%$ vs. $48.4 \%)$ in patients that had received face-to-face discussions with their PCP concerning $\mathrm{CRC}$ screening and who agreed to this type of screening.

Our study was not designed to determine overall compliance with FOBT testing, but some estimates of this can be made. During the study period, FOBT testing was ordered by PCPs in 1569 patients. Overall, 846 patients complied and received FOBT cards, which represents an overall 53.9\% compliance rate (Fig. 1). Of the 769 eligible participants, 435 (56.6\%) returned the FOBT cards, and the absolute return rate was $64.4 \%$ in patients receiving a mailed reminder and $48.4 \%$ in usual care patients. Extrapolating this to the overall rate of successful FOBT compliance in the total group for which FOBT testing was ordered, the use of a mailed reminder may increase overall FOBT compliance from 409/1569 (26.1\%) to 545/1569 (34.7\%), which again represents a relative increase of $33 \%$. Again, this presumes that FOBT testing was ordered for the purpose of CRC screening in all 1569 patients, but data are not available to verify this in all patients who did not comply with FOBT and not included in the study.

A prior review of participation in CRC screening documented overall FOBT compliance rates ranging from $10 \%$ to $50 \%$, depending on the study population, setting, and type of intervention $^{9}$. In three randomized trials, the compliance rates of selected patients that agreed to undergo FOBT testing were $59.6 \%$ to $75.2 \%^{3,4,18}$. In contrast, compliance was much lower in community based mass screening programs, ranging from $26 \%$ to $48 \%{ }^{11,12,19,20}$

Various patient interventions have been studied to increase FOBT compliance including telephone reminders, postal reminders, letters signed by personal physicians, and other educational strategies ${ }^{9}$. However, despite the most intensive strategies delivered to well-defined populations of eligible patients, overall FOBT adherence rarely increased above $50 \%{ }^{9}$. More recently, Stokamer et al. conducted a randomized, controlled trial to examine whether intense patient education from nurses would increase FOBT card return rates in U.S. Veteran patients ${ }^{11}$. The investigators were able to show that a one-on-one 10-15 minute educational session regarding the importance of CRC screening and FOBT by nurses increased FOBT completion rates to $65.9 \%$ compared with $50.1 \%$ in the control group. Despite the significant increase in FOBT completion rates, implementing this intervention would likely be expensive and time-consuming.
Prior studies of mailed reminders for improving CRC screening rates have shown mixed results, with minimal improvement documented in unselected patients ${ }^{21,22}$. More recently, although not related to FOBT adherence, Denberg et al. were able to show that a 1 page 2 -sided mailed reminder significantly increased colonoscopy appointment adherence by $11.7 \%$ and was cost-effective ${ }^{16,23}$. The study participants in Denberg's randomized controlled study were non-volunteers and were blinded to their involvement.

Limited data have been published describing patient-related factors that are associated with compliance and non-compliance with FOBT based screening. Using our diverse and large cohort, we identified several independent predictors of FOBT compliance, which include having received a mailed reminder and prior history of FOBT completion. The only negative significant factor associated with FOBT compliance was current or recent illicit drug use.

In our pooled sample, patients who had completed one, two, or three or more FOBT kits in the past were more likely to return the FOBT than those who had never completed an FOBT kit. Our observation of prior FOBT completed as a positive predictor of FOBT completion is not surprising. Patients who have completed the test in the past are more likely to be unaffected by the process of collecting their own stool samples, which has been shown to be one of the major barriers to FOBT compliance ${ }^{24}$. We also found that patients who were currently or recently using illicit drugs were $74 \%$ less likely to return the FOBT cards than non-illicit drug users. This observation again is not surprising because patients who continue to participate in risky behaviors such as illicit drug use may lack interest in their own healthcare. To our knowledge, this is the first study to show current or recent illicit drug use as a significant negative predictor of CRC screening. It is likely that illicit drug use is much more prevalent than our study reports due to the lack of systematic reporting and questioning from physicians and the refutation by patients. These data indicate that providers should take steps in addressing their patients' current or recent illicit drug use, while at the same time take extra steps to encourage CRC screening.

The strengths of our study include: the simplicity and low cost of the intervention; the large sample size; blinded, randomized control design; low exclusion criteria; long-term follow up allowing adequate time for participants to return the FOBT cards; and a comprehensive computerized medical record system to identify predictors of compliance. This study also has several limitations. First, we only enrolled patients who picked up their FOBT cards at the laboratory after their PCP visit, and do not report the overall compliance with FOBT testing ordered by PCPs. However, we have made some estimates of overall compliance as indicated above. Second, our sample was comprised of U.S. Veteran patients from San Diego, California, which limits the generalizability of our findings. For example, financial barriers for VA patients may be less than a population with lower levels of health insurance. Third, our study was unable to determine the relative degree to which compliance was influenced by the reminder itself, the patient quote regarding CRC screening, or the educational facts regarding CRC. Fourth, we did not exclude any patients over the age of 75 as recommended by the USPSTF in $2008^{5}$, because our study was performed prior to these recommendations. Lastly, current guidelines call for the use of more 
sensitive albeit more expensive fecal immunochemical tests and potentially DNA testing; however these methods of screening have not yet been widely adopted. The results of this study would be highly relevant for these tests and would help enhance the cost-effectiveness of this approach to CRC screening.

In summary, a simple, inexpensive mailed educational reminder significantly improved FOBT card return rates for CRC screening. A factor associated with FOBT noncompliance is current or recent illicit drug use; and steps to address these issues are warranted. The results of this study also indicate that further improvements in patient compliance with CRC screening are needed. Whether adding another reminder would increase patient compliance further is an important topic for future investigation. Further studies are also recommended to assess the impact of other educational or motivational techniques, such as e-mail reminder, cell phone text messaging reminders, or specific incentives for compliance with cancer screening tests.

Acknowledgements: We would like to thank Chen Mu for gathering and inputting data. We did not receive any outside funding for this project. All resources were provided by the Research Service and Department of Medicine, Veteran Affairs San Diego Healthcare System.

Conflict of Interest: None disclosed.

Corresponding Author: Samuel B. Ho, MD; Gastroenterology (111D), VA San Diego Healthcare System, 3550 La Jolla Village Drive, San Diego, CA 92161, USA (e-mail: samuel.ho2@va.gov).

\section{REFERENCES}

1. Jemal A, Siegel R, Ward E, et al. Cancer statistics, 2008. CA Cancer J Clin. 2008;58:71-96.

2. Centers for Disease Control and Prevention (CDC). Use of colorectal cancer tests - United States., 2002, 2004, and 2006. Morb Mortal Wkly Rep. 2008;57(10):253-58.

3. Hardcastle JD, Chamberlain JO, Robinson MH, et al. Randomised controlled trial of faecal-occult-blood screening for colorectal cancer. Lancet. 1996;348:1472-7.

4. Kronborg O, Fenger C, Olsen J, Jørgensen OD, Søndergaard $\mathbf{O}$ Randomised study of screening for colorectal cancer with faecal-occultblood test. Lancet. 1996;348:1467-71.

5. Screening for colorectal cancer: U. S. Preventive Services Task Force recommendation statement. Ann Intern Med. 2008;149:627-37.
6. National Comprehensive Cancer Network (NCCN). Colorectal cancer screening. Clinical Practice Guidelines in Oncology - v.1.2009. Jenkintown, PA: NCCN; 2009. Available at: http://www.nccn.org/ professionals/physician_gls/PDF/colorectal_screening.pdf Accessed July, 2009

7. Winawer S, Fletcher $\mathbf{R}, \mathbf{R e x} \mathbf{D}$, et al. Colorectal cancer screening and surveillance: clinical guidelines and rationale-Update based on new evidence. Gastroenterology. 2003;124:544-60.

8. Pignone M, Rich M, Teutsch SM, et al. Screening for colorectal cancer in adults at average risk: a summary of the evidence for the U.S. Preventive Services Task Force. Ann Intern Med. 2002;137:132-41.

9. Vernon Sw. Participation in colorectal cancer screening: a review. J Natl Cancer Inst. 1997;89:1406-22.

10. Winchester DP, Shull JH, Scanlon EF, et al. A mass screening program for colorectal cancer using chemical testing for occult blood in the stool. Cancer. 1980;45:2955-8.

11. Stokamer CL, Tenner CT, Chaudhuri J, Vazquez E, Bini EJ. Randomized controlled trial of the impact of intensive patient education on compliance with fecal occult blood testing. J Gen Intern Med. 2005;20:278-82.

12. Mahon SM. The impact of mailing fecal occult blood test kits on return rate in a community cancer screening center. Oncol Nurs Forum. 1995;22: 1259-63.

13. Wagner TH. The effectiveness of mailed patient reminders on mammography screening: a meta-analysis. Am J Prev Med. 1998;14:64-70.

14. Tseng DS, Cox E, Plane MB, Hla KM. Efficacy of patient letter reminders on cervical cancer screening: a meta-analysis. J Gen Intern Med. 2001;16:563-8.

15. Richardson JL, Mondrus GT, Danley K, Deapen D, Mack T. Impact of a mailed intervention on annual mammography and physician breast examinations among women at high risk of breast cancer. Cancer Epidemiol Biomarkers Prev. 1996;5:71-6.

16. Denberg TD, Coombes JM, Byers TE, et al. Effect of a mailed brochure on appointment-keeping for screening colonoscopy: a randomized trial. Ann Intern Med. 2006; 145:895-900.

17. The Flesch Reading Ease Readability Formula. Available at http://www. readabilityformulas.com Accessed July, 2009.

18. Mandel JS, Bond JH, Church TR, et al. Reducing mortality from colorectal cancer by screening for fecal occult blood. Minnesota Colon Cancer Control Study. N Engl J Med. 1993;328:1365-71.

19. Khubchandani IT, Karamchandani MC, Kleckner FS, et al. Mass screening for colorectal cancer. Dis Colon Rectum. 1989;32:754-8.

20. Grazzini G, Castiglione G, Isu A, et al. Colorectal cancer screening by fecal occult blood testing: results of a population-based experience. Tumori. 2000;86:384-8.

21. Thompson RS, Michnich ME, Gray J, Friedlander L, Gilson B. Maximizing compliance with hemoccult screening for colon cancer in clinical practice. Med Care1986:24:904-14.

22. Myers RE, Ross EA, Wolf TA, Balshem A, Jepson C, Millner L. Behavioral interventions to increase adherence in colorectal cancer screening. Med Care. 1991;29:1039-50.

23. Shankaran V, McKoy JM, Dandade N, et al. Costs and cost-effectiveness of a low-intensity patient-directed intervention to promote colorectal cancer screening. J Clin Oncol. 2007 Nov 20;25:5248-53.

24. Ahlquist DA. Occult blood screening. Obstacles to effectiveness. Cancer. 1992;70:1259-65. 